\section{Accidental Acute Cannabis Intoxication Presenting as Seizure in Pediatrics Patients}

Jessica Emoto, B.A. ${ }^{1}$, Kerri Weeks, M.D. ${ }^{1}$,

K. James Kallail, Ph.D. ${ }^{2}$

University of Kansas School of Medicine-Wichita, Wichita, KS

'Department of Pediatrics

${ }^{2}$ Department of Internal Medicine

Received Jan. 22, 2020; Accepted for publication March 5, 2020; Published online May 21, 2020

\section{INTRODUCTION}

Cannabis is becoming more available as it is legalized across the country. ${ }^{1}$ There has been a rise in accidental pediatric cannabis poisoning in Colorado since its legalization. ${ }^{2}$ While recreational use of cannabis has not been legalized in the state of Kansas, its proximity to Colorado makes cannabis easily available.

Children aged one to four years of age are especially at risk for accidental poisoning due to exploratory ingestion. ${ }^{3}$ With cannabis's growing popularity, its modes of delivery have evolved to include vaping, dabbing (inhaling small quantities of a concentrated or vaporized drug), topical applications, and oral ingestion through baked goods and candies. ${ }^{4}$ Oral ingestion puts children particularly at risk of acute intoxication due to the appealing nature of edibles and its easy consumption. Furthermore, delta-9-tetrahydrocannabinol (THC) often is highly concentrated in edibles. When smoked, the maximum plasma concentration of THC is achieved within 0.02-0.5 hours versus 1-3.4 hours with oral consumption. ${ }^{5}$

THC is the main psychoactive ingredient in cannabis that contributes to its CNS toxicities. ${ }^{6}$ Cannabidiol (CBD) is the main non-psychoactive ingredient in cannabis that is being studied for its effectiveness in treating epilepsy. Through selective breeding of the hemp plant Cannabis sativa, the THC to cannabidiol content in cannabis has increased from 14 times in 1995 to about 80 times in $2014 .^{7}$

The adverse effects of cannabis intoxication in adults has been well-defined, ${ }^{8}$ but less is known about how it presents in children. There have been different case reports of acute cannabis intoxication in children presenting with ataxia, lethargy, tremors, coma, and seizures. ${ }^{8,9}$ Neurological impairment is the main manifestation of cannabis intoxication in children under 3 -years-old. ${ }^{10}$ However, the pharmacokinetics of cannabis in children is still unknown. ${ }^{11}$ Case reports depicting these side effects have dated back to 1983 in Italy ${ }^{12}$ and 2005 in the United States. ${ }^{2}$

We report two cases of children presenting with new-onset seizures precipitated by acute cannabis intoxication. Physicians should consider cannabis ingestion in their differential diagnosis when a child presents with seizures and other neurological impairments.

\section{CASE REPORT}

Case 1. A previously healthy 3-year-old male was admitted for care of a new-onset seizure. The patient's mother reported that the child climbed in bed with her on the morning of admission complaining of a headache and dizziness. He had a generalized seizure for 30 - 45 seconds and was postictal afterward. A minor head trauma three days prior to admission was noted by the mother, but it was noncontributory to the presentation.
KANSAS JOURNAL of MEDIC INE

The child was a term baby without significant complications. According to the mother, he had normal growth and development. He had asthma and allergies, for which he took albuterol and cetirizine as needed. He was up-to-date on his immunizations and had no known drug allergies, prior hospitalizations, or surgeries. The child had routine visits to a primary care physician and dental care.

The mother reported no family history of seizures and the extended family history was not significant for any childhood illnesses or diseases. The mother had depression, anxiety, and Borderline Personality Disorder. The patient was living with his mother, her female friend, and the friend's two small children. The mother denied any domestic violence or possible substances in the home. The mother adamantly denied any cannabis in the home.

The patient was given levetiracetam on admission. Physical exam was normal and he was developmentally appropriate and neurologically back to baseline. No further seizures occurred. Before drug testing was conducted, rectal diazepam was prescribed as needed for future episodes. A head computed tomography (CT) scan and brain magnetic resonance imaging (MRI) were unremarkable. Urinalysis was negative and electroencephalogram (EEG) was normal. A urine drug screen (UDS) as part of the initial workup for new-onset seizure was positive for cannabis. A quantitative urine toxicology test showed THC levels were $750 \mathrm{ng} / \mathrm{mL}$, which is the highest the test would read. Other drugs, ethanol, acetaminophen, and salicylates were negative.

After investigation, the source of THC exposure could not be determined. However, since the levels were high, the intoxication was unlikely to be from passive smoke inhalation and likely from THClaced edibles. Ultimately, the lack of history to identify the source of exposure prompted concern for lack of supervision and safety of the child. The family was referred to Kansas Department for Children and Families (DCF).

Case 2. A 3-year-old female was admitted by emergency medical services (EMS) with complaints of altered mental status, lethargy, vomiting, and seizure activity. The parents reported that the child was at the mother's house in the days leading up to admission. The child took a nap as usual, but she was unresponsive when the mother went to check on her. When EMS arrived, the patient was lethargic, vomiting, and seizing. Her Glasgow Coma Score was 6 at the scene.

The child had no prior hospitalizations, surgeries, or injuries. She had normal growth, development, and behavior. The mother's family was significant for a history of seizures.

A head CT was negative, and a brain MRI and ECG were normal. The patient's UDS was negative for acetaminophen and salicylate and positive for THC and benzodiazepines. The benzodiazepines were given by EMS for the seizures. Confirmatory urine testing was completed with maximum levels of THC reported.

When questioned about the positive THC drug screen, the mother reported that she had been to Colorado recently and brought back THC-containing brownies and other edibles. The mother claimed 
KANSAS JOURNAL of MEDICINE ACUTE CANNABIS INTOXICATION PRESENTING AS SEIZURE continued.

to have discarded the edibles and denied any knowledge of the child ingesting any THC-containing foods. The mother denied regular use of any substances.

The child improved overnight and was clinically approved for discharge. The Kansas DCF was alerted and followed up with the family.

\section{DISCUSSION}

With the increasing availability of cannabis products, acute intoxication from accidental ingestion should be considered when a child presents with new-onset seizure. While the mode of cannabis intoxication was not determined in the first case, the high levels of THC and age of the child suggested oral ingestion. The child had experienced a minor head trauma three days prior to admission, and while concussion was ruled out, the trauma could have been a contributing factor to the new-onset seizure.

THC-containing products may lower the seizure threshold in a predisposed individual with an associated family history, ${ }^{2}$ such as with the patient in the second case. However, the pediatric pharmacokinetics of THC have not been well established. A recent study found the mean peak plasma concentration of THC extract to be two to seven hours and elimination half-life to be 4.0 hours in pediatric patients, but this was conducted in a small population $(\mathrm{n}=9)$ with a median age of 11 years and controlled dosing. ${ }^{13}$ In adults, THC is detectable in plasma for over one week, and its metabolites can persist in feces and urine for several weeks. ${ }^{14}$ Obtaining quantitative levels of THC in plasma and urine or feces can help determine temporality in a patient with an unknown exposure.

In the past, cannabis use has not been associated with causing the onset of seizures. We postulate this is due to the lower concentrations of THC typically found in cannabis products until recent years. Due to the increasing use of THC-containing products, physicians should consider a toxicology screen including common drugs of abuse in pediatric patients presenting with new-onset seizure. Upon any positive screen, a quantitative serum test also should be ordered to assist the diagnosis. Treatment of acute cannabis intoxication is mainly supportive care; an accurate diagnosis can avoid unnecessary tests and interventions. Our report emphasized including acute cannabis intoxication in the differential diagnosis of pediatric patients at risk (one to four years of age) of accidental ingestion who present with new-onset seizure.

\section{REFERENCES}

${ }^{1}$ Shi Y, Liang D, Bao Y, An R, Wallace MS, Grant I. Recreational marijuana legalization and prescription opioids received by Medicaid enrollees. Drug Alcohol Depend 2019; 194:13-19. PMID: 30390550.

${ }^{2}$ Wang GS, Le Lait MC, Deakyne SJ, Bronstein AC, Bajaj L, Roosevelt G. Unintentional pediatric exposures to marijuana in Colorado, 2009-2015. JAMA Pediatr 2016; 170(9):el60971. PMID: 27454910.

3 Mofenson HC, Greensher J. The unknown poison. Pediatrics 1974; 54(3):336-342. PMID: 4153526.

4 Johnson RM, Brooks-Russell A, Ma M, Fairman BJ, Tolliver RL Jr, Levinson AH. Usual modes of marijuana consumption among high school students in Colorado. J Stud Alcohol Drugs 2016; 77(4):580-588. PMID: 27340962.
${ }^{5}$ Cox EJ, Maharao N, Patilea-Vrana G, et al. A marijuana-drug interaction primer: Precipitants, pharmacology, and pharmacokinetics. Pharmacol Ther 2019; 201:25-38. PMID: 31071346.

${ }^{6}$ Fasinu PS, Philips S, EISohly MA, Walker LA. Current status and prospects for cannabidiol preparations as new therapeutic agents. Pharmacotherapy 2016; 36(7):781-796. PMID: 27285147.

7 ElSohly MA, Mehmedic Z, Foster S, Gon C, Chandra S, Church JC. Changes in cannabis potency over the last 2 decades (1995-2014): Analysis of current data in the United States. Biol Psychiatry 2016; 79(7):613-619. PMID: 26903403.

${ }^{8}$ Grotenhermen F. Pharmacokinetics and pharmacodynamics of cannabinoids. Clin Pharmacokinet 2003; 42(4):327-360. PMID: 12648025.

9 Bonkowsky JL, Sarco D, Pomeroy SL. Ataxia and shaking in a 2-year-old girl: Acute marijuana intoxication presenting as seizure. Pediatr Emerg Care 2005; $21(8): 527-528$. PMID: 16096599.

${ }^{10}$ Claudet I, Le Breton M, Bréhin C, Franchitto N. A 10-year review of cannabis exposure in children under 3-years of age: Do we need a more global approach? Eur J Pediatr 2017; 176(4):553-556. PMID: 28210835.

${ }^{11}$ Huntsman RJ, Tang-Wai R, Schackelford AE. Cannabis for pediatric epilepsy. J Clin Neurophysiol 2020; 37(1):2-8. PMID: 31895184.

12 Malizia E, Andreucci G, Alfani F, Smeriglio M, Nicholai P. Acute intoxication with nicotine alkaloids and cannabinoids in children from ingestion of cigarettes. Hum toxicol 1983; 2(2):315-316. PMID: 6862475.

${ }^{13}$ Wang GS, Bourne DWA, Klawitter J, et al. Disposition of oral delta-9 tetrahydrocannabinol (THC) in children receiving cannabis extracts for epilepsy. Clin toxicol 2020; 58(2):124-128. PMID: 31111749.

${ }^{14}$ McGilveray IJ. Pharmacokinetics of cannabinoids. Pain Res Manag 2005; 10(Suppl A):15A-22A. PMID: 16237477.

Keywords: cannabis, substance-related disorders, seizure, pediatrics, case report 